\title{
Repositioning of the Mental Nerve in Case of Severe Mandibular Atrophy: A Case Series
}

\author{
${ }^{1}$ Merve Nur Kadioglu, ${ }^{2}$ Mehmet Fatih Senturk, ${ }^{3}$ Aysegül Mine Tuzuner Oncul, ${ }^{4}$ Mine Cambazoglu
}

\begin{abstract}
Following tooth extraction, cortical bone suffers greater resorption on the vestibular area and patients typically present narrow and low alveolar crests. In case of moderate to severe mandibular atrophy; the bone height between alveolar crest and mental nerve is too narrow and patients may feel pain under pressure. Repositioning of the mental nerve is an effective preprosthetic surgery procedure in patients with hyperesthesia caused by dental prosthesis. As well as this surgical repositioning of the mental nerve facilitates the placement of implants can prevent pain, in overdenture prosthesis and fixed dentures. In this report, mental nerve repositioning, implant placement in the interforaminal region of the mandible and prosthetic rehabilitation of two cases with hyperesthesia due to severe mandibular atrophy is presented. Nerve repositioning may constitute a treatment alternative in patients with a consequent risk of mental nerve damage during the placement of dental implants.
\end{abstract}

Keywords: Transposition of mental nerve, Implants, Overdenture prosthesis.

How to cite this article: Kadioglu MN, Senturk MF, Oncul AMT, Cambazoglu M. Repositioning of the Mental Nerve in Case of Severe Mandibular Atrophy: A Case Series. Int J Experiment Dent Sci 2014;3(1):41-43.

\section{Source of support: Nil}

Conflict of interest: None declared

\section{INTRODUCTION}

Severely athrophic mandibles comprise a challenge for the oral and maxillofacial surgeons as well as the prosthodontists. ${ }^{1}$ For these patients different implant based rehabilitation techniques can be used. For this purpose several procedures have been suggested, such as alveolar distraction, bone grafting and nerve repositioning. ${ }^{2}$

Transposition of the mental nerve is a preprosthetic procedure that is effective for patients with hyperesthesia caused

\footnotetext{
${ }^{1}$ Resident, ${ }^{2}$ Assistant Professor, ${ }^{3}$ Associate Professor ${ }^{4}$ Professor

${ }^{1,3,4}$ Department of Oral and Maxillofacial Surgery, Ankara University, Ankara, Turkey

${ }^{2}$ Department of Oral and Maxillofacial Surgery, Suleyman Demirel University, Isparta,Turkey
}

Corresponding Author: Merve Nur Kadioglu, Resident Department of Oral and Maxillofacial Surgery, Ankara University, Ankara, Turkey, e-mail: mnkadioglu@gmail.com by the pressure effect of a dental prosthesis on the alveolar ridge. The first case of mental nerve repositioning was published in 1967 by Pasqual and Pasqual. ${ }^{3}$ It is also performed to protect the location for implant insertion and to preserve the inferior alveolar nerve during mandibular tumor excision surgery. ${ }^{4}$ Although, nerve repositioning is a complex procedure, with a high risk of sensory disturbances (anesthesia, paresthesia or neuralgia, mostly of a transient nature, but sometimes permanent) there is a risk of nerve injury through primary surgical trauma or secondary complications, such as edema or hematoma. ${ }^{2,5,6}$ According to Thoma ${ }^{7}$ extensive atrophy of the alveolar process of the mandible may bring the ridge down to the mental foramen. This is usually seen in geriatric patients. These patients often report severe pain and losing the use of prosthesis. Stimulated symptoms can be produced by digital pressure on the mental foramen area. In many cases relief of the prosthesis on the remaining denture-bearing area can correct the problem. A conservative surgical procedure for the correction of this condition is repositioning of the mental nerve. ${ }^{3,4}$

In these reports we present two cases of severe mandibular atrophy in which mental nerve repositioning, implant placement and prosthetic rehabilitation were carried out. Nerve repositioning is a treatment alternative in patients with a consequent risk of mental nerve damage during the placement of dental implants.

\section{CASE PRESENTATIONS}

\section{Case 1}

A 66-year-old female patient referred to the Department of Oral and Maxillofacial Surgery, Faculty of Dentistry, University of Ankara with a chief complaint of pain in the right mental foreman area and inability to use her denture. The patient's medical history revealed that she used partial dentures for a long time. The clinical examination showed, severe mandibular atrophy. The preoperative panoramic radiographic analysis showed that; position off the mental foramen was nearby the alveolar crest (Fig. 1). Consequently mental nerve repositioning was decided with implant placement on both canine sides in the same operation. Under local anesthesia and sedoanalgesia, crestal incision was planned and mucoperiosteal flap was removed. Mental nerve was dissected from the buccal flap carefully. A distal and caudal 


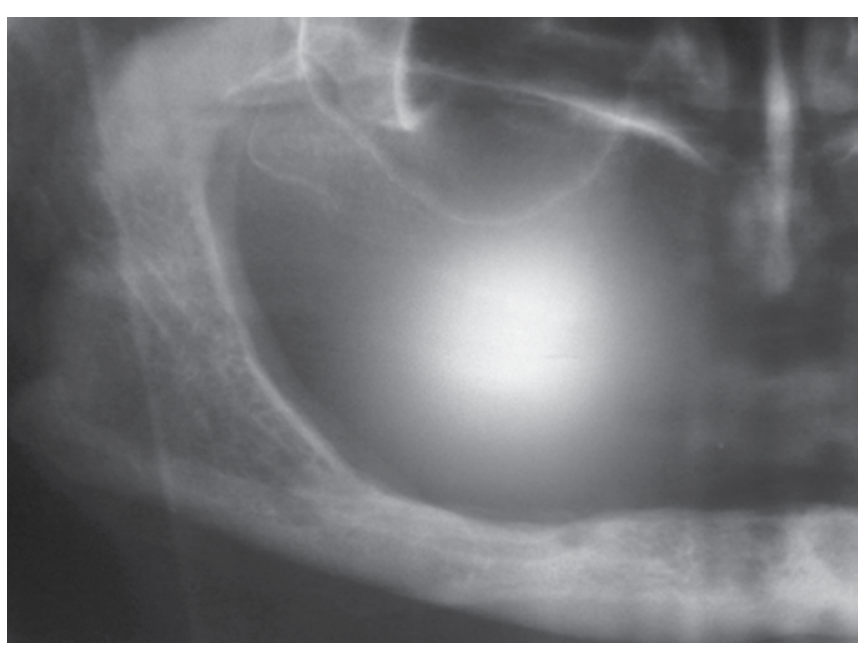

Fig. 1: Preoperative panoramic radiographic view of the patient

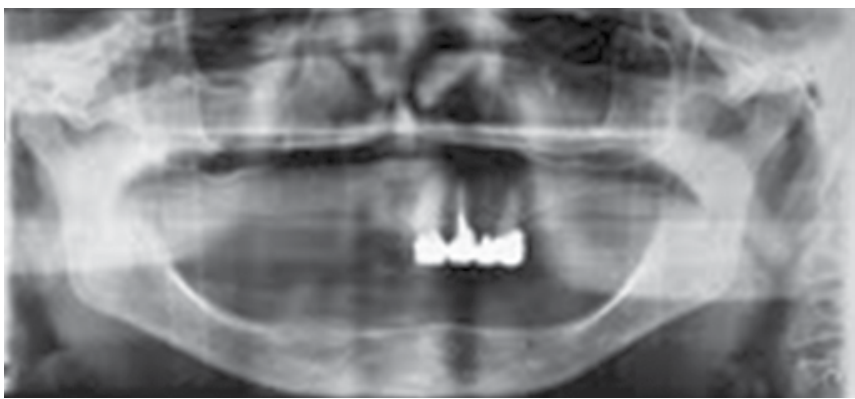

Fig. 3: Preoperative panoramic radiographic view

transposition of the mental nerve trunk was done with piezosurgery instruments. Careful dissection of the connective tissue off the nerve trunk was done so that the foramen could be identified. After removing approximately $6 \mathrm{~mm}$ posterior-inferior cortical bone, mental nerve was transposed inferiorly to the mental foramen (Fig. 2) and posterior bone gap was packed with bone wax immediately after, two endosseous implants (Zimmer dental implant) were placed in the inter-foraminal area. The flap was sutured with 3-0 vicryl (Johnson and Johnson). Neurosensory function of the mental nerve was assessed objectively. There was hypoesthesia of the mental nerve on the first postoperative day and there was no pain in the mental foramen area. Healing was uneventful and the neurosensory test 3 weeks after transposition showed normal function of the mental nerve. After the 6 months of the operation, the patient began to implant overdenture prosthesis contently.

\section{Case 2}

A 54-year-old patient referred to the Department of Oral and Maxillofacial Surgery, Faculty of Dentistry, University of Ankara with a chief complaint of pain in the right mental foreman area and inability to use her denture. Clinical and radiographic examination showed that the patient had severe mandibular atrophy and mandibular foramen was too close to the alveolar crest (Fig. 3). Consequently bilateral mental

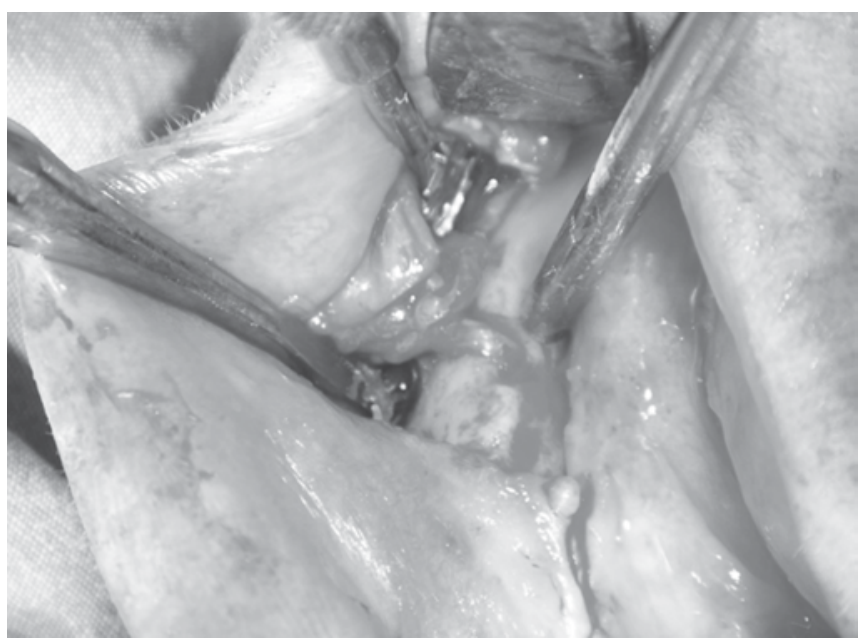

Fig. 2: Intraoperative view

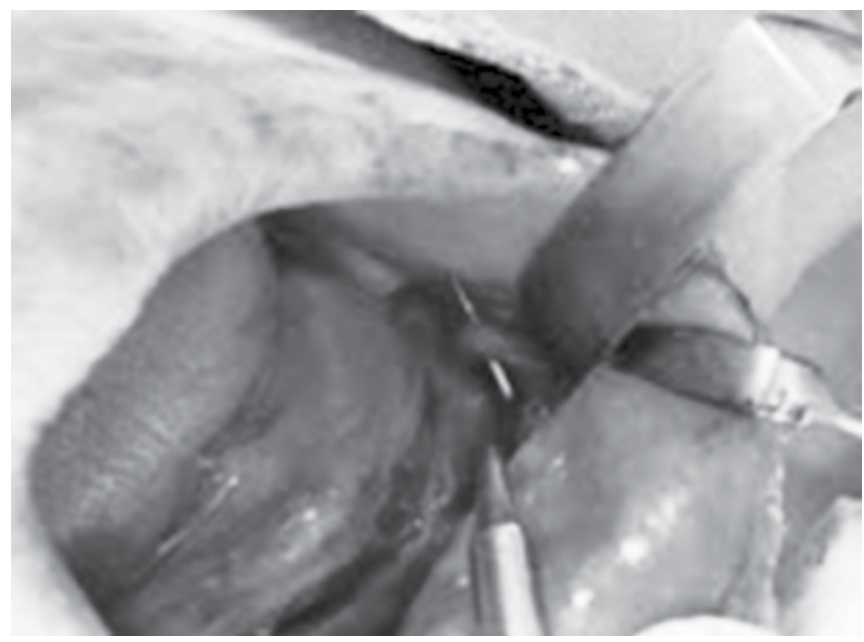

Fig. 4: Intraoperative view shown the nerve trunk

nerve repositioning was decided with implant placement on both canine sides in the same surgical step. Under local anesthesia and sedoanalgesia, crestal incision was planned and mucoperiosteal flap was reflected. Mental nerve dissected from the buccal flap rigorously. A distal and inferior transposition of the mental nerve trunk was done with controversial bur instrument. Careful dissection of the connective tissue off the nerve trunk was done so that the foramen could be identified (Fig. 4). After removing approximately $7 \mathrm{~mm}$ posterior-inferior cortical bone, mental nerve was transposed inferiorly to the mental foramen and posterior bone gap was packed with bone wax immediately. After the two endosseous implants (Zimmer) were placed in the inter-foraminal area. Finally the flap was sutured with 3-0 vicryl. Postoperatively, the patient was assessed for neurosensory dysfunction, pain, or other complications. There was hypoesthesia of the mental nerve on the first postoperative day and there was no pain. Healing was uneventful and the neurosensory test 4 weeks after transposition showed normal function. After 6 months of the implant placement, the patient began use to the implant overdenture prosthesis contently. 


\section{DISCUSSION}

Transposition of the mental nerve trunk is performed as a preprosthetic procedure. It is effective for patients with hyperesthesia caused by dental prosthesis and resorption of the alveolar ridge caused by aging. ${ }^{8-11}$ This is a procedure in which the bone is usually cut with rotating instruments and piezosurgery techniques. $3,4,9,12$

Although there have been several reports about inferior alveolar nerve transposition, ${ }^{1,2,5}$ there are only few reports presenting the mental nerve transpositioning. 3,4

Metzger and colleagues ${ }^{6}$ reported that in vitro preparation with the piezoelectric device was more invasive to the bone than with the conventional diamond bur. They also reported that the degree and risk of injury with the piezoelectric device was lower than with the conventional rotary bur. ${ }^{6}$ Despite extreme caution, injures caused by compression or direct trauma by the rotating instrument on nerve tissue cannot be excluded. ${ }^{9,12}$ In our cases we performed the nerve transposition with the help of piezosurgery in one case, in another case with steel bur. Both of the cases postoperative 6 months follow-up was uneventful and pain symptoms in both cases were reliefed.

Kale and colleagues ${ }^{4}$ mobilized the nerve $8 \mathrm{~mm}$ distally and $7 \mathrm{~mm}$ inferiorly keeping in mind a scope for further vestibuloplasties also the incisive branch was sacrificed as the patient was edentulous and the nerve supplies only the lower anterior teeth. ${ }^{4}$ However, Sakkas et $\mathrm{al}^{9}$ did only a caudal transposition of the right mental nerve trunk using piezosurgery under local anesthesia. After 3 months, two dental implants were placed to the interforaminal area of the mandible and 3 months later mandibular overdenture prosthesis was inserted. ${ }^{9}$ In our both cases we transposed the mental nerve distally and inferiorly like Kale et $\mathrm{al}^{4}$ and totally four implants (2-2) placed in the interforaminal region of the mandible at the same operation. Three months later overdentures were inserted.

Nerve repositioning may constitute a treatment alternative in patients with a consequent risk of mental nerve damage during the placement of dental implants. Undoubtedly a careful operating technique remains critical because soft tissue could be injured by excessive mechanical force from the tip of the instrument. Careful nerve manipulation and mobilization is required in order to reduce the risk of neurosensory alterations. Despite the possible complications; the technique is usually well-tolerated by the patients. ${ }^{3,4}$

\section{REFERENCES}

1. Jensen J, Reiche-Fischel O, Sindet-Pedersen S. Nerve transposition and implant placement in the atrophic posterior mandibular alveolar ridge. J Oral Maxillofac Surg 1994;52(7):662-668.

2. Del Castillo Pardo De Vera JL, Chamorro Pons M, Cebrian Carretero JL. Reposioning of the inferior alveolar nerve in cases of severe mandibular atrophy: a clinical case. Med Oral Patol Oral Cir Bucal 2008;13(12):E778-782.

3. Pasqual RJ, Pasqual HN. Surgical repositioning of the mental nerve. Report of a case. Oral Surg Oral Med Oral Pathol 1967; 24(3):305-306.

4. Kale TP, Patel JN, Bhutani H. Mental nerve reposioning: a case report. Int J Dent Clin 2010;2(3):58-60.

5. Nocini PF, De Santis D, Fracasso E, Zanette G. Clinical and electrophysiological assessment of inferior alveolar nerve function after lateral nerve transposition. Clin Oral Implants Res 1999; 10(2):120-130.

6. Metzger MC, Bormann KH, Schoen R, Gellrich NC, Schmelzeisen $M$. Inferior alveolar nerve transposition an in vitro comparison between piezosurgery and conventional bur use. J Oral Implantol 2006;32(1):19-25.

7. Thoma KH. Oral surgery. 2nd ed. St Louis, Miss: CV Mosby 1952;1.

8. Shibahara T, Noma H, Yamane G, Katakura A, Takasaki Y, Tamada Y. Transposition of the mental nerve and inferior alveolar nerve trunk. Bull Tokyo Dent Coll 1996;37(2):103-107.

9. Sakkas N, Otten JE, Gutwald R, Schmelzeisen R. Transposition of the mental nerve by piezosurgery followed by postoperative neurosensory control: a case report. Br J Oral Maxillofac Surg 2008;46(4):270-271.

10. di Lauro F, Testa NF, Gentile E. Avitabile A. Lowering of the mental nerve. Stomatol Mediterr 1988;8(4):339-342.

11. Mathis H. Surgical measures for preparing the oral cavity for dental prosthesis. Zahnarztl Rundsch 1954;63(7):186-194.

12. Lambrecht JT. Intraoral piezosurgery. Schweiz Monatsschr Zahnmed 2004;114(1):28-36. 